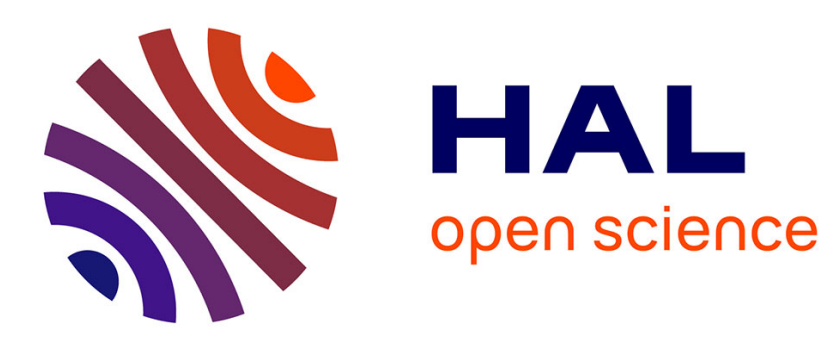

\title{
Hybrid High-Order methods for variable diffusion problems on general meshes
}

\author{
Daniele Antonio Di Pietro, Alexandre Ern
}

\section{To cite this version:}

Daniele Antonio Di Pietro, Alexandre Ern. Hybrid High-Order methods for variable diffusion problems on general meshes. Comptes Rendus. Mathématique, 2014, 353, pp.31-34. 10.1016/j.crma.2014.10.013 . hal-01023302

\section{HAL Id: hal-01023302 \\ https://hal.science/hal-01023302}

Submitted on 11 Jul 2014

HAL is a multi-disciplinary open access archive for the deposit and dissemination of scientific research documents, whether they are published or not. The documents may come from teaching and research institutions in France or abroad, or from public or private research centers.
L'archive ouverte pluridisciplinaire HAL, est destinée au dépôt et à la diffusion de documents scientifiques de niveau recherche, publiés ou non, émanant des établissements d'enseignement et de recherche français ou étrangers, des laboratoires publics ou privés. 


\title{
Hybrid High-Order Methods for Variable-Diffusion Problems on General Meshes
}

\author{
Daniele A. Di Pietro ${ }^{\mathrm{a}}$, Alexandre Ern $^{\mathrm{b}}$ \\ ${ }^{a}$ University Montpellier 2, I3M, 34057 Montpellier Cedex 5, France \\ ${ }^{b}$ University Paris-Est, CERMICS (ENPC), 77455 Marne la Vallée Cedex 2, France
}

\begin{abstract}
We extend the Hybrid High-Order method introduced by the authors for the Poisson problem to problems with heterogeneous/anisotropic diffusion. The cornerstone is a local discrete gradient reconstruction from element- and face-based polynomial degrees of freedom. Optimal error estimates are proved.
\end{abstract}

\section{Résumé}

Méthodes hybrides d'ordre élevé pour des problèmes à diffusion variable sur des maillages généraux. Nous étendons la méthode hybride d'ordre élevé conçue par les auteurs pour le problème de Poisson à des problèmes de diffusion hétérogène/anisotrope. La pierre angulaire est une reconstruction locale du gradient discret à partir des degrés de liberté polynomiaux sur les éléments et les faces. On établit des estimations d'erreur optimales.

\section{Introduction}

Let $\Omega \subset \mathbb{R}^{d}, d \in\{2,3\}$, denote an open, bounded, polytopic domain. Let $f \in L^{2}(\Omega)$ and, for a subset $X \subset \bar{\Omega}$, denote by $(\cdot, \cdot)_{X}$ and $\|\cdot\|_{X}$ the inner product and norm in $L^{2}(X)$, respectively. We focus on the following variable-diffusion problem: Find $u \in U_{0}:=H_{0}^{1}(\Omega)$ such that

$$
(\kappa \nabla u, \nabla v)_{\Omega}=(f, v)_{\Omega} \quad \forall v \in U_{0},
$$

where $\boldsymbol{\kappa}$ is a bounded, tensor-valued function in $\Omega$, taking symmetric values with lowest eigenvalue uniformly bounded from below away from zero. Owing to the Lax-Milgram Lemma, problem (1) is well-posed.

The approximation of diffusive problems on general polytopic meshes has received an increasing attention lately. Several low-order methods have been developed; see, e.g., [1, 2] and references therein. Recently, high-order methods have also become available; we mention the high-order Mimetic Finite Difference (MFD) schemes [3, 4, the Virtual Element Method [5], the Mixed High-Order method [6], and the Hybrid HighOrder (HHO) methods 7, 8. For the latter, the degrees of freedom (DOFs) are scalar-valued polynomials at mesh elements and faces up to some degree $k \geq 0$ (as for the MFD schemes in 4]), and the construction hinges on (i) a local discrete gradient reconstruction of order $k$ and (ii) a least-squares local penalty that weakly enforces the matching between element- and face-based DOFs while preserving the order of the gradient reconstruction. This design leads to optimal energy- and $L^{2}$-norm error estimates; cf. [7] for the Poisson problem ( $\boldsymbol{\kappa}$ being the identity tensor in (1) ) and 8 for (quasi-incompressible) linear elasticity.

The purpose of the present work is to extend the HHO method of [7] to the variable-diffusion problem (1). The key idea is to modify the gradient reconstruction so as to account for the diffusion tensor $\boldsymbol{\kappa}$. Then, adapting the ideas of [7, we prove stability of the discrete problem and derive optimal error estimates. We make the reasonable assumption that there is a partition $P_{\Omega}$ of $\Omega$ so that $\boldsymbol{\kappa}$ is piecewise Lipschitz. For simplicity of exposition, we also assume that $\boldsymbol{\kappa}$ is a piecewise polynomial; otherwise, an additional quadrature error has to be accounted for. In applications from the geosciences, $\boldsymbol{\kappa}$ can often be taken piecewise constant.

Email addresses: daniele.di-pietro@univ-montp2.fr (Daniele A. Di Pietro), ern@cermics.enpc.fr (Alexandre Ern) 


\section{Discrete setting and local gradient reconstruction}

We consider admissible mesh sequences in the sense of [9, Sect. 1.4]. Each mesh $\mathcal{T}_{h}$ in the sequence is a finite collection $\{T\}$ of nonempty, disjoint, open, polytopic elements such that $\bar{\Omega}=\bigcup_{T \in \mathcal{T}_{h}} \bar{T}$ and $h=\max _{T \in \mathcal{T}_{h}} h_{T}$ (with $h_{T}$ the diameter of $T$ ), and there is a matching simplicial submesh of $\mathcal{T}_{h}$ with locally equivalent mesh size and which is shape-regular in the usual sense. For all $T \in \mathcal{T}_{h}$, the faces of $T$ are collected in the set $\mathcal{F}_{T}$. In an admissible mesh sequence, $\operatorname{card}\left(\mathcal{F}_{T}\right)$ is uniformly bounded, the usual discrete and multiplicative trace inequalities hold on element faces, and the $L^{2}$-orthogonal projector onto polynomial spaces enjoys optimal approximation properties on each mesh element. Let a polynomial degree $k \geq 0$ be fixed. For all $T \in \mathcal{T}_{h}$, we define the local space of DOFs as $U_{T}^{k}:=\mathbb{P}_{d}^{k}(T) \times\left\{\times_{F \in \mathcal{F}_{T}} \mathbb{P}_{d-1}^{k}(F)\right\}$, where $\mathbb{P}_{d}^{k}(T)$ (resp., $\mathbb{P}_{d-1}^{k}(F)$ ) is spanned by the restrictions to $T$ (resp., $\left.F\right)$ of $d$-variate (resp., $(d-1)$-variate) polynomials of total degree $\leq k$. In what follows, $A \lesssim B$ denotes the inequality $A \leq C B$ with positive constant $C$ independent of the polynomial degree $k$, the meshsize $h$, and the diffusion tensor $\boldsymbol{\kappa}$. We assume that each mesh $\mathcal{T}_{h}$ in the sequence is compatible with the partition $P_{\Omega}$ associated with the diffusion tensor. We denote by $\kappa_{T}^{b}$ and $\kappa_{T}^{\sharp}$ the lowest and largest eigenvalue of $\boldsymbol{\kappa}$ in $T$, respectively, and we introduce the local heterogeneity/anisotropy ratio $\rho_{T}:=\kappa_{T}^{\sharp} / \kappa_{T}^{\mathrm{b}} \geq 1$. In what follows, we explicitly track the dependency of the bounds on the ratio $\rho_{T}$. To avoid the profileration of symbols, we assume that for all $T \in \mathcal{T}_{h}$, the Lipschitz constant of $\kappa$ in $T$, say $L_{T}^{\kappa}$, satisfies $L_{T}^{\kappa} \lesssim \kappa_{T}^{\sharp}$.

For all $T \in \mathcal{T}_{h}$, we define the local gradient reconstruction operator $\boldsymbol{G}_{T}^{k}: \mathrm{U}_{T}^{k} \rightarrow \boldsymbol{\nabla} \mathbb{P}_{d}^{k+1}(T)$ such that, for all $\vee:=\left(\mathrm{v}_{T},\left(\mathrm{v}_{F}\right)_{F \in \mathcal{F}_{T}}\right) \in \mathrm{U}_{T}^{k}$ and all $w \in \mathbb{P}_{d}^{k+1}(T)$,

$$
\left(\boldsymbol{\kappa} \boldsymbol{G}_{T}^{k} \mathrm{v}, \boldsymbol{\nabla} w\right)_{T}=\left(\boldsymbol{\kappa} \nabla \mathrm{v}_{T}, \boldsymbol{\nabla} w\right)_{T}+\sum_{F \in \mathcal{F}_{T}}\left(\mathrm{v}_{F}-\mathrm{v}_{T}, \boldsymbol{\nabla} w \cdot \boldsymbol{\kappa} \cdot \boldsymbol{n}_{T F}\right)_{F},
$$

which can be computed by solving a local (well-posed) Neumann problem in $\mathbb{P}_{d}^{k+1}(T)$. We next introduce the potential reconstruction operator $p_{T}^{k}: \mathrm{U}_{T}^{k} \rightarrow \mathbb{P}_{d}^{k+1}(T)$ such that, for all $\vee \in \mathrm{U}_{T}^{k}, \nabla p_{T}^{k} \vee:=G_{T}^{k} \vee$ and $\int_{T} p_{T}^{k} \mathrm{v}:=\int_{T} \mathrm{v}_{T}\left(p_{T}^{k} \mathrm{v}\right.$ is well-defined since $\left.\boldsymbol{G}_{T}^{k} \vee \in \mathbb{\nabla}_{d}^{k+1}(T)\right)$. Finally, we define the local interpolation operator $\mathbf{I}_{T}^{k}: H^{1}(T) \rightarrow \mathrm{U}_{T}^{k}$ such that, for all $v \in H^{1}(T), \mathrm{I}_{T}^{k} v:=\left(\pi_{T}^{k} v,\left(\pi_{F}^{k} v\right)_{F \in \mathcal{F}_{T}}\right)$, where $\pi_{T}^{k}$ and $\pi_{F}^{k}$ are the $L^{2}$-orthogonal projectors onto $\mathbb{P}_{d}^{k}(T)$ and $\mathbb{P}_{d-1}^{k}(F)$, respectively.

Lemma 2.1 (Approximation properties for $\left.p_{T}^{k}\right|_{T} ^{k}$ ). The following holds for all $v \in H^{k+2}(T)$ with $\alpha=1 / 2$ if $\boldsymbol{\kappa}$ is piecewise constant and $\alpha=1$ in the general case:

$$
\left\|v-\left.p_{T}^{k}\right|_{T} ^{k} v\right\|_{T}+h_{T}^{1 / 2}\left\|v-\left.p_{T}^{k}\right|_{T} ^{k} v\right\|_{\partial T}+h_{T}\left\|\nabla\left(v-p_{T}^{k} l_{T}^{k} v\right)\right\|_{T}+h_{T}^{3 / 2}\left\|\nabla\left(v-\left.p_{T}^{k}\right|_{T} ^{k} v\right)\right\|_{\partial T} \lesssim \rho_{T}^{\alpha} h_{T}^{k+2}\|v\|_{H^{k+2}(T)} .
$$

Proof. Let $v \in H^{k+2}(T)$. A direct calculation using (2), the definitions of $p_{T}^{k}$ and $\mathbf{I}_{T}^{k}$, and integration by parts shows that, for all $w \in \mathbb{P}_{d}^{k+1}(T)$,

$$
\left(\boldsymbol{\kappa} \boldsymbol{\nabla}\left(v-\left.p_{T}^{k}\right|_{T} ^{k} v\right), \boldsymbol{\nabla} w\right)_{T}=\left(\left(\boldsymbol{\kappa}-\overline{\boldsymbol{\kappa}}_{T}\right) \boldsymbol{\nabla}\left(v-\pi_{T}^{k} v\right), \boldsymbol{\nabla} w\right)_{T}-\sum_{F \in \mathcal{F}_{T}}\left(\pi_{F}^{k} v-\pi_{T}^{k} v, \boldsymbol{\nabla} w \cdot\left(\boldsymbol{\kappa}-\overline{\boldsymbol{\kappa}}_{T}\right) \cdot \boldsymbol{n}_{T F}\right)_{F},
$$

where $\overline{\boldsymbol{\kappa}}_{T}$ denotes the mean-value of $\boldsymbol{\kappa}$ in $T$. Note that the right-hand side vanishes if $\boldsymbol{\kappa}$ is piecewise constant. In the general case, owing to the assumptions on $\boldsymbol{\kappa}$ and using the approximation properties of the $L^{2}$-orthogonal projectors along with a discrete trace inequality for $\left\|\boldsymbol{\kappa}^{1 / 2} \nabla w\right\|_{F}$, we infer that

$$
\left|\left(\boldsymbol{\kappa} \nabla\left(v-\left.p_{T}^{k}\right|_{T} ^{k} v\right), \boldsymbol{\nabla} w\right)_{T}\right| \lesssim L_{T}^{\kappa} h_{T} h_{T}^{k}\|v\|_{H^{k+1}(T)}\|\boldsymbol{\nabla} w\|_{T} \lesssim \kappa_{T}^{\sharp} h_{T}^{k+1}\|v\|_{H^{k+1}(T)}\|\boldsymbol{\nabla} w\|_{T} .
$$

We now observe that

$$
\left\|\boldsymbol{\kappa}^{1 / 2} \boldsymbol{\nabla}\left(v-\left.p_{T}^{k}\right|_{T} ^{k} v\right)\right\|_{T}^{2}=\left(\boldsymbol{\kappa} \boldsymbol{\nabla}\left(v-\left.p_{T}^{k}\right|_{T} ^{k} v\right), \boldsymbol{\nabla}\left(v-\pi_{T}^{k+1} v\right)\right)_{T}+\left(\boldsymbol{\kappa} \boldsymbol{\nabla}\left(v-\left.p_{T}^{k}\right|_{T} ^{k} v\right), \boldsymbol{\nabla}\left(\pi_{T}^{k+1} v-\left.p_{T}^{k}\right|_{T} ^{k} v\right)\right)_{T} .
$$

Denote by $\mathfrak{T}_{1}$ and $\mathfrak{T}_{2}$ the addends on the right-hand side of (5). Using the Cauchy-Schwarz inequality and the approximation properties of $\pi_{T}^{k+1}$, we obtain $\left|\mathfrak{T}_{1}\right| \lesssim\left\|\boldsymbol{\kappa}^{1 / 2} \nabla\left(v-p_{T}^{k} T_{T}^{k} v\right)\right\|_{T}\left(\kappa_{T}^{\sharp}\right)^{1 / 2} h_{T}^{k+1}\|v\|_{H^{k+2}(T)}$. 
When $\boldsymbol{\kappa}$ is piecewise constant, $\mathfrak{T}_{2}$ vanishes, so that using Young's inequality yields $\left\|\boldsymbol{\nabla}\left(v-\left.p_{T}^{k}\right|_{T} ^{k} v\right)\right\|_{T} \leq$ $\left(\kappa_{T}^{b}\right)^{-1 / 2}\left\|\boldsymbol{\kappa}^{1 / 2} \nabla\left(v-\left.p_{T}^{k}\right|_{T} ^{k} v\right)\right\|_{T} \lesssim \rho_{T}^{1 / 2} h_{T}^{k+1}\|v\|_{H^{k+2}(T)}$. In the general case, using (4) with $w=\left(\pi_{T}^{k+1} v-\left.p_{T}^{k}\right|_{T} ^{k} v\right)$ and since $\left\|\boldsymbol{\nabla}\left(\pi_{T}^{k+1} v-\left.p_{T}^{k}\right|_{T} ^{k} v\right)\right\|_{T}=\left\|\boldsymbol{\nabla} \pi_{T}^{k+1}\left(v-\left.p_{T}^{k}\right|_{T} ^{k} v\right)\right\|_{T} \lesssim\left\|\nabla\left(v-\left.p_{T}^{k}\right|_{T} ^{k} v\right)\right\|_{T}$ owing to the $H^{1}$-stability of the projector $\pi_{T}^{k+1}$, we infer that $\left|\mathfrak{T}_{2}\right| \lesssim \rho_{T}^{1 / 2}\left(\kappa_{T}^{\sharp}\right)^{1 / 2} h_{T}^{k+1}\|v\|_{H^{k+1}(T)}\left\|\boldsymbol{\kappa}^{1 / 2} \nabla\left(v-\left.p_{T}^{k}\right|_{T} ^{k} v\right)\right\|_{T}$, which leads to the estimate on $\left\|\boldsymbol{\nabla}\left(v-\left.p_{T}^{k}\right|_{T} ^{k} v\right)\right\|_{T}$ in (3). The other terms in (3) are then bounded as in [7, Lemma 3].

Remark $1(\alpha=0)$. It is also possible to take $\alpha=0$ whenever, for all $T \in \mathcal{T}_{h}$, the eigenvectors of $\boldsymbol{\kappa}_{\mid T}$ are constant and its eigenvalues satisfy, with obvious notation, $\left|\lambda(x)-\bar{\lambda}_{T}\right| \lesssim h_{T} \lambda(x)$ for all $x \in T$.

\section{Discrete problem and stability}

For all $T \in \mathcal{T}_{h}$, we introduce the local bilinear forms $a_{T}$ and $s_{T}$ on $\mathrm{U}_{T}^{k} \times \mathrm{U}_{T}^{k}$ such that

$$
a_{T}(\mathrm{u}, \mathrm{v}):=\left(\kappa \boldsymbol{G}_{T}^{k} \mathrm{u}, \boldsymbol{G}_{T}^{k} \mathrm{v}\right)_{T}+s_{T}(\mathrm{u}, \mathrm{v}), \quad s_{T}(\mathrm{u}, \mathrm{v}):=\sum_{F \in \mathcal{F}_{T}} \frac{\kappa_{F}}{h_{F}}\left(\pi_{F}^{k}\left(\mathrm{u}_{F}-P_{T}^{k} \mathrm{u}\right), \pi_{F}^{k}\left(\mathrm{v}_{F}-P_{T}^{k} \mathrm{v}\right)\right)_{F},
$$

with $\kappa_{F}:=\left\|\boldsymbol{n}_{T F} \cdot \boldsymbol{\kappa} \cdot \boldsymbol{n}_{T F}\right\|_{L^{\infty}(F)}$ and the local potential reconstruction $P_{T}^{k}: \mathrm{U}_{T}^{k} \rightarrow \mathbb{P}_{d}^{k+1}(T)$ such that $P_{T}^{k} \mathrm{v}:=\mathrm{v}_{T}+\left(p_{T}^{k} \mathrm{v}-\pi_{T}^{k} p_{T}^{k} \mathrm{v}\right)$. We define the global space of DOFs by patching interface values, so that $\mathrm{U}_{h}^{k}:=\left\{\times_{T \in \mathcal{T}_{h}} \mathbb{P}_{d}^{k}(T)\right\} \times\left\{\times_{F \in \mathcal{F}_{h}} \mathbb{P}_{d-1}^{k}(F)\right\}$, and, for all $T \in \mathcal{T}_{h}$, we denote by $\mathrm{L}_{T}: \mathrm{U}_{h}^{k} \rightarrow \mathrm{U}_{T}^{k}$ the restriction operator that maps the global DOFs in $\mathrm{U}_{h}^{k}$ to the corresponding local DOFs in $\mathrm{U}_{T}^{k}$. The discrete problem consists in seeking $\mathrm{u}_{h} \in \mathrm{U}_{h, 0}^{k}:=\left\{\mathrm{v}_{h}=\left(\left(\mathrm{v}_{T}\right)_{T \in \mathcal{T}_{h}},\left(\mathrm{v}_{F}\right)_{F \in \mathcal{F}_{h}}\right) \in \mathrm{U}_{h}^{k} \mid \mathrm{v}_{F} \equiv 0 \forall F \in \mathcal{F}_{h}^{\mathrm{b}}\right\}$ such that

$$
a_{h}\left(\mathrm{u}_{h}, \mathrm{v}_{h}\right):=\sum_{T \in \mathcal{T}_{h}} a_{T}\left(\mathrm{~L}_{T} \mathrm{u}_{h}, \mathrm{~L}_{T} \mathrm{v}_{h}\right)=\sum_{T \in \mathcal{T}_{h}}\left(f, \mathrm{v}_{T}\right)_{T}=: l_{h}\left(\mathrm{v}_{h}\right) \quad \forall \mathrm{v}_{h} \in \mathrm{U}_{h, 0}^{k} .
$$

To analyze the stability of the discrete problem, we introduce the following seminorm on $\mathrm{U}_{T}^{k}$ :

$$
\|\mathrm{v}\|_{\boldsymbol{\kappa}, T}^{2}:=\left\|\boldsymbol{\kappa}^{1 / 2} \nabla \mathrm{v}_{T}\right\|_{T}^{2}+\sum_{F \in \mathcal{F}_{T}} \frac{\kappa_{F}}{h_{F}}\left\|\mathrm{v}_{F}-\mathrm{v}_{T}\right\|_{F}^{2},
$$

and we set $\left\|\mathrm{v}_{h}\right\|_{\kappa, h}^{2}:=\sum_{T \in \mathcal{T}_{h}} \rho_{T}^{-1}\left\|\mathrm{~L}_{T} \mathrm{v}_{h}\right\|_{\kappa, T}^{2}$ for all $\mathrm{v}_{h} \in \mathrm{U}_{h}^{k}$. Observe that $\|\cdot\|_{\kappa, h}$ is a norm on $\mathrm{U}_{h, 0}^{k}$.

Lemma 3.1 (Stability). The following inequalities hold for all $\vee \in \mathrm{U}_{T}^{k}$ :

$$
\rho_{T}^{-1}\|\mathrm{v}\|_{\boldsymbol{\kappa}, T}^{2} \lesssim a_{T}(\mathrm{v}, \mathrm{v}) \lesssim \rho_{T}\|\mathrm{v}\|_{\boldsymbol{\kappa}, T}^{2}
$$

Consequently, $\left\|\mathrm{v}_{h}\right\|_{\kappa, h}^{2} \lesssim a_{h}\left(\mathrm{v}_{h}, \mathrm{v}_{h}\right)$ for all $\mathrm{v}_{h} \in \mathrm{U}_{h}^{k}$ and problem 47 is well-posed.

Proof. We adapt the proof of [7, Lemma 4]. Concerning the face terms, we obtain

$$
\sum_{F \in \mathcal{F}_{T}} \frac{\kappa_{F}}{h_{F}}\left\|\mathrm{v}_{F}-\mathrm{v}_{T}\right\|_{F}^{2} \leq s_{T}(\mathrm{v}, \mathrm{v})+\rho_{T}\left\|\boldsymbol{\kappa}^{1 / 2} \boldsymbol{G}_{T}^{k} \mathrm{v}\right\|_{T}^{2}, \quad s_{T}(\mathrm{v}, \mathrm{v}) \lesssim \sum_{F \in \mathcal{F}_{T}} \frac{\kappa_{F}}{h_{F}}\left\|\mathrm{v}_{F}-\mathrm{v}_{T}\right\|_{F}^{2}+\rho_{T}\left\|\boldsymbol{\kappa}^{1 / 2} \boldsymbol{G}_{T}^{k} \mathrm{v}\right\|_{T}^{2} .
$$

To compare $\left\|\boldsymbol{\kappa}^{1 / 2} \boldsymbol{G}_{T}^{k} \mathrm{v}\right\|_{T}$ and $\left\|\boldsymbol{\kappa}^{1 / 2} \nabla \vee_{T}\right\|_{T}$, we observe that, for all $w \in \mathbb{P}_{d}^{k+1}(T)$ and all $F \in \mathcal{F}_{T}$,

$$
\left\|\boldsymbol{\nabla} w \cdot \boldsymbol{\kappa} \cdot \boldsymbol{n}_{T F}\right\|_{F}^{2} \leq\left(\left|\boldsymbol{n}_{T F} \cdot \boldsymbol{\kappa} \cdot \boldsymbol{n}_{T F}\right|,|\boldsymbol{\nabla} w \cdot \boldsymbol{\kappa} \cdot \boldsymbol{\nabla} w|\right)_{F} \lesssim \frac{\kappa_{F}}{h_{F}}\left\|\boldsymbol{\kappa}^{1 / 2} \boldsymbol{\nabla} w\right\|_{T}^{2},
$$

where we have used the Cauchy-Schwarz inequality for $\kappa$, the definition of $\kappa_{F}$, and a discrete trace inequality. Taking $w=\mathrm{v}_{T}$ in the definition (2) of $\boldsymbol{G}_{T}^{k} \mathrm{v}$ yields $\left\|\boldsymbol{\kappa}^{1 / 2} \nabla \mathrm{v}_{T}\right\|_{T}^{2}=\left(\boldsymbol{\kappa} \boldsymbol{G}_{T}^{k} \mathrm{v}, \boldsymbol{\nabla} \mathrm{v}_{T}\right)_{T}-\sum_{F \in \mathcal{F}_{T}}\left(\mathrm{v}_{F}-\right.$ $\left.\mathrm{v}_{T}, \boldsymbol{\nabla} \mathbf{v}_{T} \cdot \boldsymbol{\kappa} \cdot \boldsymbol{n}_{T F}\right)_{F}$. Hence, using (11), a discrete trace inequality for $\left\|\boldsymbol{\kappa}^{1 / 2} \nabla \mathbf{v}_{T}\right\|_{F}$, the first bound in (10), $\rho_{T} \geq 1$, and Young's inequality yields

$$
\left\|\boldsymbol{\kappa}^{1 / 2} \nabla \mathrm{v}_{T}\right\|_{T}^{2} \lesssim\left\|\boldsymbol{\kappa}^{1 / 2} \boldsymbol{G}_{T}^{k} \mathrm{v}\right\|_{T}^{2}+\sum_{F \in \mathcal{F}_{T}} \frac{\kappa_{F}}{h_{F}}\left\|\mathrm{v}_{F}-\mathrm{v}_{T}\right\|_{F}^{2} \lesssim \rho_{T}\left\|\boldsymbol{\kappa}^{1 / 2} \boldsymbol{G}_{T}^{k} \mathrm{v}\right\|_{T}^{2}+s_{T}(\mathrm{v}, \mathrm{v})
$$

Moreover, since $\left\|\boldsymbol{\kappa}^{1 / 2} \boldsymbol{G}_{T}^{k} \mathrm{v}\right\|_{T}=\sup _{w \in \mathbb{P}_{d}^{k+1}(T)} \frac{\left(\boldsymbol{\kappa} \boldsymbol{G}_{T}^{k} \mathrm{v}, \boldsymbol{\nabla} w\right)_{T}}{\left\|\boldsymbol{\kappa}^{1 / 2} \boldsymbol{\nabla} w\right\|_{T}}$ and proceeding similarly leads to $\left\|\boldsymbol{\kappa}^{1 / 2} \boldsymbol{G}_{T}^{k} \mathrm{v}\right\|_{T} \lesssim$ $\|v\|_{\kappa, T}$. Combining the above bounds yields (9), and the rest of the proof is straightforward. 


\section{Error analysis}

Theorem 4.1 (Energy-error estimate). Let $u \in U_{0}$ solve (1) and let $\mathrm{u}_{h} \in \mathrm{U}_{h, 0}^{k}$ solve (7). Assume that $u_{\mid T} \in H^{k+2}(T)$ for all $T \in \mathcal{T}_{h}$. Then, letting $\widehat{\mathrm{u}}_{h}:=\left(\left(\pi_{T}^{k} u\right)_{T \in \mathcal{T}_{h}},\left(\pi_{F}^{k} u\right)_{F \in \mathcal{F}_{h}}\right) \in \mathrm{U}_{h, 0}^{k}$ and, recalling the definition of $\alpha$ from Lemma 2.1, the following holds with consistency error $\mathcal{E}_{h}\left(\mathrm{v}_{h}\right):=a_{h}\left(\widehat{\mathrm{u}}_{h}, \mathrm{v}_{h}\right)-l_{h}\left(\mathrm{v}_{h}\right)$ :

$$
\left\|\widehat{\mathrm{u}}_{h}-\mathrm{u}_{h}\right\|_{\boldsymbol{\kappa}, h} \lesssim \sup _{\mathrm{v}_{h} \in \mathrm{U}_{h, 0}^{k},\left\|\mathrm{v}_{h}\right\|_{\boldsymbol{\kappa}, h}=1} \mathcal{E}_{h}\left(\mathrm{v}_{h}\right) \lesssim\left\{\sum_{T \in \mathcal{T}_{h}} \kappa_{T}^{\sharp} \rho_{T}^{1+2 \alpha} h_{T}^{2(k+1)}\|u\|_{H^{k+2}(T)}^{2}\right\}^{1 / 2} .
$$

Proof. We adapt the proof of [7, Theorem 8]. The first inequality in $[12$ is an immediate consequence of Lemma 3.1. Proceeding as in [7] with $\check{u}_{T}:=p_{T}^{k} \mathrm{~L}_{T} \widehat{\mathrm{u}}_{h}=p_{T}^{k} \mathrm{l}_{T}^{k}\left(u_{\mid T}\right)$ and $\mathrm{v}_{h} \in \mathrm{U}_{h, 0}^{k}$ with $\left\|\mathrm{v}_{h}\right\|_{\kappa, h}=1$ leads to

$$
\mathcal{E}_{h}\left(\mathrm{v}_{h}\right)=\sum_{T \in \mathcal{T}_{h}}\left(\boldsymbol{\kappa} \nabla\left(\check{u}_{T}-u\right), \nabla \mathbf{v}_{T}\right)_{T}+\sum_{T \in \mathcal{T}_{h}} \sum_{F \in \mathcal{F}_{T}}\left(\mathrm{v}_{F}-\mathrm{v}_{T},\left(\boldsymbol{\nabla} \check{u}_{T}-\boldsymbol{\nabla} u\right) \cdot \boldsymbol{\kappa} \cdot \boldsymbol{n}_{T F}\right)_{F}+\sum_{T \in \mathcal{T}_{h}} s_{T}\left(\mathrm{~L}_{T} \widehat{\mathbf{u}}_{h}, \mathrm{~L}_{T} \mathbf{v}_{h}\right) .
$$

Denote by $\mathfrak{T}_{1}, \mathfrak{T}_{2}, \mathfrak{T}_{3}$ the three terms on the right-hand side. Combining the results of Lemmas 2.1 and 3.1. we infer that $\left|\mathfrak{T}_{1}+\mathfrak{T}_{2}\right|^{2} \lesssim \sum_{T \in \mathcal{T}_{h}} \kappa_{T}^{\sharp} \rho_{T}^{1+2 \alpha} h_{T}^{2(k+1)}\|u\|_{H^{k+2}(T)}^{2}$. Moreover, since $s_{T}\left(\mathrm{~L}_{T} \widehat{\mathrm{u}}_{h}, \mathrm{~L}_{T} \mathrm{v}_{h}\right) \leq$ $s_{T}\left(\mathrm{~L}_{T} \widehat{\mathrm{u}}_{h}, \mathrm{~L}_{T} \widehat{\mathrm{u}}_{h}\right)^{1 / 2} s_{T}\left(\mathrm{~L}_{T} \mathrm{v}_{h}, \mathrm{~L}_{T} \mathrm{~V}_{h}\right)^{1 / 2}$, proceeding as in 7 for the first factor, and using the second bound in 10. for the second factor yields $\left|\mathfrak{T}_{3}\right|^{2} \lesssim \sum_{T \in \mathcal{T}_{h}} \kappa_{T}^{\sharp} \rho_{T}^{1+2 \alpha} h_{T}^{2(k+1)}\|u\|_{H^{k+2}(T)}^{2}$.

Finally, adapting the proof of [7, Theorem 10] leads to the following $L^{2}$-norm error estimate.

Theorem 4.2 ( $L^{2}$-error estimate). Assume elliptic regularity for problem (1) in the form $\|z\|_{H^{2}(\Omega)} \lesssim\|g\|_{\Omega}$ for all $g \in L^{2}(\Omega)$ and $z \in U_{0}$ solving (1) with data $g$. Assume $f \in H^{k+\delta}(\Omega)$ with $\delta=0$ for $k \geq 1$ and $\delta=1$ for $k=0$. Then, using the same notation as in Theorem 4.1. and defining the piecewise polynomial functions $\widehat{u}_{h}$ and $u_{h}$ such that $\widehat{u}_{h \mid T}=\pi_{T}^{k} u$ and $u_{h \mid T}=u_{T}$ for all $T \in \mathcal{T}_{h}$, the following holds:

$$
\left\|\widehat{u}_{h}-u_{h}\right\|_{\Omega} \lesssim\left|\left(\kappa^{\sharp}\right)^{1 / 2} \rho^{1 / 2+\alpha} h\right|_{\ell^{\infty}}\left\{\sum_{T \in \mathcal{T}_{h}} \kappa_{T}^{\sharp} \rho_{T}^{1+2 \alpha} h_{T}^{2(k+1)}\|u\|_{H^{k+2}(T)}^{2}\right\}^{1 / 2}+h^{k+2}\|f\|_{H^{k+\delta}(\Omega)},
$$

where $\left|\left(\kappa^{\sharp}\right)^{1 / 2} \rho^{1 / 2+\alpha} h\right|_{\ell \infty}:=\max _{T \in \mathcal{T}_{h}}\left(\kappa_{T}^{\sharp}\right)^{1 / 2} \rho_{T}^{1 / 2+\alpha} h_{T}$.

\section{References}

[1] J. Droniou, R. Eymard, T. Gallouët, R. Herbin, A unified approach to mimetic finite difference, hybrid finite volume and mixed finite volume methods, M3AS Mathematical Models and Methods in Applied Sciences 20 (2) (2010) 265-295.

[2] J. Bonelle, A. Ern, Analysis of compatible discrete operator schemes for elliptic problems on polyhedral meshes, M2AN Math. Model. Numer. Anal. 48 (2) (2014) 553-581.

[3] L. Beirão da Veiga, K. Lipnikov, G. Manzini, Arbitrary-order nodal mimetic discretizations of elliptic problems on polygonal meshes, SIAM J. Numer. Anal. 49 (5) (2011) 1737-1760.

[4] G. Manzini, K. Lipnikov, A high-order mimetic method on unstructured polyhedral meshes for the diffusion equation, J. Comput. Phys.Published online. DOI 10.1016/j.jcp.2014.04.021

[5] L. Beirão da Veiga, F. Brezzi, A. Cangiani, G. Manzini, L. D. Marini, A. Russo, Basic principles of virtual element methods, M3AS Math. Models Methods Appl. Sci. 23 (1) (2013) 199-214.

[6] D. A. Di Pietro, A. Ern, A family of arbitrary-order mixed methods for heterogeneous anisotropic diffusion on general meshes, Submitted. Preprint hal-00918482 (2014).

[7] D. A. Di Pietro, A. Ern, S. Lemaire, An arbitrary-order and compact-stencil discretization of diffusion on general meshes based on local reconstruction operators, Comput. Methods Appl. Math.Published online. DOI 10.1515/cmam-2014-0018.

[8] D. A. Di Pietro, A. Ern, A hybrid high-order locking-free method for linear elasticity on general meshes, Submitted. Preprint hal-00979435 (2014).

[9] D. A. Di Pietro, A. Ern, Mathematical aspects of discontinuous Galerkin methods, Vol. 69 of Mathématiques \& Applications, Springer-Verlag, Berlin, 2012. 